\title{
Semiclassical theory of transport in antidot lattices*
}

\author{
Gregor Hackenbroich and Felix von Oppen \\ Max-Planck-Institut für Kernphysik, $6911^{17}$ Heidelberg, Germany
}

(October 28, 2018)

\begin{abstract}
Motivated by a recent experiment by Weiss et al. [Phys. Rev. Lett. 70, 4118 (1993)], we present a detailed study of quantum transport in large antidot arrays whose classical dynamics is chaotic. We calculate the longitudinal and Hall conductivities semiclassically starting from the Kubo formula. The leading contribution reproduces the classical conductivity. In addition, we find oscillatory quantum corrections to the classical conductivity which are given in terms of the periodic orbits of the system. These periodic-orbit contributions provide a consistent explanation of the quantum oscillations in the magnetoconductivity observed by Weiss et al. We find that the phase of the oscillations with Fermi energy and magnetic field is given by the classical action of the periodic orbit. The amplitude is determined by the stability and the velocity correlations of the orbit. The amplitude also decreases exponentially with temperature on the scale of the inverse orbit traversal time $\hbar / T_{\gamma}$. The Zeeman splitting leads to beating of the amplitude with magnetic field. We also present an analogous semiclassical derivation of Shubnikov-de Haas oscillations where the corresponding classical motion is integrable. We show that the quantum oscillations in antidot lattices and the Shubnikov-de Haas oscillations are closely related. Observation of both effects requires that the
\end{abstract}

\footnotetext{
${ }^{*}$ This paper is dedicated to Prof. H. Wagner on the occasion of his 60th birthday.
} 
elastic and inelastic scattering lengths be larger than the lengths of the relevant periodic orbits. The amplitude of the quantum oscillations in antidot lattices is of a higher power in Planck's constant $\hbar$ and hence smaller than that of Shubnikov-de Haas oscillations. In this sense, the quantum oscillations in the conductivity are a sensitive probe of chaos.

73.50.Jt, 03.65.Sq, 05.45.+b

Typeset using REVTEX 


\section{INTRODUCTION}

Over the past few years high-mobility semiconductor heterostructures have proved to be a valuable tool for studying the implications of quantum chaos experimentally [1]. Advances in technology have made it possible to manufacture structures whose elastic and inelastic mean free paths exceed the relevant sample dimensions. In these ballistic (micro)structures essentially all large-angle scattering is due to the geometric features of the device [2]. Hence, they can be used in particular for experimental studies of billiard systems [3] 7] which have been central to the theoretical development of quantum chaos [1]. These novel experimental realizations of quantum-chaotic systems are important because there are actually only a small number of systems in the field of quantum chaos that allow for a controlled comparison between theory and experiment.

Much of the work on quantum chaos has focused on the spectral properties of systems whose classical dynamics is chaotic [1]. However, the detailed spectrum is not an accessible observable in ballistic semiconductor structures. Rather, one measures thermodynamic properties such as the magnetic susceptibility [8] and the persistent current [9] or transport properties such as the conductance [3 [7] of the sample. Hence the challenge to theory was whether these quantities are sensitive to the nature of the underlying classical dynamics. This question has now been answered in the affirmative for both thermodynamic and transport properties. With regard to transport properties of ballistic microstructures, it has been predicted that the power spectrum of the conductance fluctuations [10,11] and the lineshape of the weak-localization peak [11,12] are probes of quantum chaos and there is accumulating experimental evidence in favor of these predictions [4,6,7]. There are also predictions that the amplitudes of the persistent current [13] and the magnetization [14, 15] are sensitive probes of quantum chaos. These have not yet been subject to experimental tests.

Chaotic transport in semiconductor structures has been investigated in two kinds of experiments. Experiments on mesoscopic ballistic cavities with the shape of chaotic billiards coupled to leads [4,6,7] have focused on generic effects such as conductance fluctuations 
and weak localization. These effects are familiar from the study of transport in diffusive conductors [16]. In the other type of experiment one measures magnetotransport in macroscopic antidot lattices. Antidot lattices consist of a periodic array of holes "drilled" into a two-dimensional electron gas [3,5:19]. They are macroscopic in the sense that the phase-coherence length is much smaller than the system size (the lattice spacing of the antidot array, on the other hand, may be smaller than the phase-coherence length) [21]. Antidot lattices can be viewed as an approximate experimental realization of the Sinai billiard [20] which is a well-known chaotic system. Experiments on antidot arrays were mostly concerned with features in the magnetotransport which reflect the specific nature of the sample. In this paper we present a theoretical study of transport in antidot lattices [22].

Two analytical methods dominated theoretical studies of quantum chaos, random-matrix theory [23] and the semiclassical approximation [24]. While the first is most appropriate for describing generic effects due to long trajectories, semiclassical theory is especially useful in describing effects due to short system-specific trajectories. We find that semiclassical theory is particularly suited to address the system-specific features in the magnetoconductivity of antidot lattices. In the context of quantum chaos interest in semiclassical theory derives mostly from the fact that, strictly speaking, chaos is a concept of classical physics. Semiclassical theory allows one to study most explicitly the implications of (classical) chaos in quantum mechanics. In the context of ballistic structures semiclassical theory is a natural approach because the Fermi wavelength is often the shortest length scale in the problem. Furthermore, the contributions of long classical trajectories are frequently suppressed by finite temperature or inelastic scattering, rendering the semiclassical expressions quite manageable 13 15.

The first experiments on antidot lattices by Weiss et al. [3] focused on the regime of classical transport. They revealed a series of pronounced magnetoresistance peaks at magnetic fields where the classical cyclotron radius is commensurate with the lattice spacing of the antidot array. For these magnetic-field values, the electrons can circle around a certain number of antidots for long times. This effectively slows them down leading to an increase 
in the resistivity $3,25,26$. Here we are concerned with more recent experiments at lower temperatures [5] which revealed additional quantum oscillations in the magnetoresistance superimposed on the classical peaks. The period of these oscillations corresponds to roughly a flux quantum threading a unit cell of the antidot lattice. Depending on the sample these oscillations varied between $1 / B$ periodicity familiar from Shubnikov-de Haas oscillations [27] and $B$ periodicity familiar from Aharonov-Bohm oscillations [28]. Weiss et al. [5] explained these observations phenomenologically by assuming that the resistivity is proportional to the square of the density of states, $\rho_{x x} \sim d^{2}\left(E_{F}, B\right)$. The period of the oscillations can then be understood in terms of a few short periodic orbits which, according to Gutzwiller's trace formula [24], give oscillatory contributions to the density of states. This approach is clearly unsatisfactory because it does not make any predictions concerning the absolute amplitude of the oscillations and, more importantly, because this relation between the resistivity and the density of states cannot be derived from first principles. In fact, this relation certainly does not reproduce the pronounced classical peaks in the resistivity 229.

Nevertheless, the apparent success of the phenomenological theory of Ref. [5] suggested that there is indeed a relation between quantum corrections to transport properties and classical periodic orbits. In this paper we present a semiclassical theory for the conductivity starting from first principles [30]. Since we are considering macroscopic antidot lattices much larger than elastic and inelastic mean free paths we start from the Kubo formula. To leading order in Planck's constant $\hbar$ we reproduce the classical Kubo formula. According to Fleischmann et al. [25] this contribution accounts for the classical peaks in the resistivity. The central result of this paper is that we find quantum corrections to the classical conductivity which explain the additional quantum oscillations observed by Weiss et al. [5]. These quantum contributions to the conductivity are indeed given in terms of classical periodic orbits. The contribution of each orbit oscillates as function of Fermi energy and magnetic field with a phase equal to its classical action. The amplitude depends on the stability of and velocity correlations along the orbit. The predictions of our theory for the period, the amplitude, and the temperature dependence of the periodic-orbit contributions 
are consistent with the experimental results of Weiss et al. [5]. It was previously known that periodic orbits are important in describing the oscillatory corrections to the density of states [24] and hence for thermodynamic properties of mesoscopic systems [31, 13, 15, 32, 33]. Our results combined with the experimental results of Ref. [5] show that periodic orbits are also important for quantum corrections of transport properties [34]. There have also been attempts to interpret the "frequency spectrum" of conductance fluctuations in billiard-shaped ballistic microstructures in terms of periodic orbits [4,21]. The relevance of our results to these experiments is not clear because the conductance of such phase-coherent samples is more appropriately described using the Landauer-Büttiker approach rather than the Kubo formula.

We find that the quantum oscillations in the conductivity of "chaotic" antidot lattices are closely related to the Shubnikov-de Haas oscillations in the conductivity of the "classically integrable" two-dimensional electron gas. To make this relation explicit we present an analogous semiclassical derivation of Shubnikov-de Haas oscillations. Even though both effects are closely related, the technical treatment is somewhat different. It is well known that integrable and chaotic systems must be treated differently in the semiclassical theory of the spectral density [24]. Likewise we find that certain spatial integrals which must be computed exactly in the derivation of Shubnikov-de Haas oscillations are evaluated by the stationary-phase method for chaotic antidot lattices. As a result, the amplitude of Shubnikov-de Haas oscillations is larger than that of the quantum oscillatons in antidot lattices. In analogy to the semiclassical theory of the spectral density [24], our results for antidot lattices are strictly valid only if the classical dynamics is completely chaotic. In section $\$ 1 \mathrm{C}$ we present evidence that this is indeed the case to a good approximation for the main sample investigated in Ref. [5].

The outline of the paper is as follows. In section $\llbracket$ we present the semiclassical theory for Shubnikov-de Haas oscillations. Section [II] contains the central results of this paper on the longitudinal conductivity ([IIA) and the Hall conductivity ([IB) of antidot lattices, and a comparison between our theory and the experimental results of Ref. [5] ([II Q). Various 
details of the calculations in sections [I], [IIA, and ШIIB are given in three appendices. Finally, we conclude in section IV.

\section{SHUBNIKOV-DE HAAS OSCILLATIONS}

In this section we present a semiclassical derivation of the Shubnikov-de Haas oscillations in the magnetoconductivity of two-dimensional electron gases. Our motivation for doing so is twofold. First, it is instructive to see how the semiclassical methods which we employ in our derivation of the quantum oscillations in antidot lattices work in a more familiar context. Second, it turns out that the Shubnikov-de Haas and the quantum oscillations in antidot lattices are closely related phenomena. This relationship is brought out explicitly by comparing the calculations for both effects.

Throughout this paper we consider transport in macroscopic systems in the sense that the sample dimensions are much larger than the elastic and inelastic mean free paths. In this regime, transport is appropriately described by the Kubo formula. Here, we start from the Kubo formula for the longitudinal conductivity

$$
\sigma_{x x}=\frac{e^{2} \pi \hbar}{\Omega} \operatorname{Tr}\left\{\hat{v}_{x} \delta_{\Gamma}\left(E_{F}-\hat{H}\right) \hat{v}_{x} \delta_{\Gamma}\left(E_{F}-\hat{H}\right)\right\}
$$

where $\hat{H}=(1 / 2 m)(\hat{\mathbf{p}}-e \mathbf{A}(\hat{\mathbf{r}}))^{2}$ is the Hamiltonian of a two-dimensional electron gas in a perpendicular magnetic field, $\mathbf{A}=(-B y / 2, B x / 2)$ is the vector potential taken in the symmetric gauge, and $\hat{v}_{x}$ is the $x$ component of the velocity operator (here $m$ denotes the effective mass of the electrons). The Fermi energy is denoted by $E_{F}$ and the volume of the system by $\Omega$. It is well known that one needs to include some disorder scattering when directly computing the dc conductivity within the Kubo formalism 35. We include weak disorder at the level of the Born approximation by giving the $\delta$-functions a finite width $\Gamma=\hbar / 2 \tau_{e l}\left(\tau_{e l}\right.$ is the elastic scattering time). This is sufficient to prove our main point, i.e., that there is a precise connection between classical periodic orbits and quantum corrections to the conductivity. However, it should be kept in mind that the Born approximation cannot 
fully describe smooth disorder potentials because it does not distinguish between the elastic and the transport mean free paths. Unfortunately, it appears to be a difficult problem to go beyond the Born approximation when using the semiclassical approach presented in this paper. In principle, the level broadening $\Gamma$ also includes a contribution due to inelastic scattering. This will be left implicit throughout the paper.

To evaluate the Kubo formula (11), we start by expressing the $\delta$-functions in Eq. (11) in terms of the retarded and advanced Green functions $\hat{G}^{ \pm}(E)=[E-\hat{H} \pm i \Gamma]^{-1}$,

$$
\delta_{\Gamma}\left(E_{F}-\hat{H}\right)=-\frac{1}{2 \pi i}\left[\hat{G}^{+}\left(E_{F}\right)-\hat{G}^{-}\left(E_{F}\right)\right] .
$$

Evaluating the trace in (11) in position representation, one finds that the longitudinal conductivity can be expressed as

$$
\sigma_{x x}=\sigma_{x x}^{++}+\sigma_{x x}^{--}+\sigma_{x x}^{+-},
$$

where

$$
\sigma_{x x}^{ \pm \pm}=-\frac{e^{2} \pi \hbar}{\Omega}\left(\frac{1}{2 \pi m}\right)^{2} \int d^{2} r d^{2} r^{\prime}\left[\left(\frac{\hbar}{i} \nabla_{x}-e A_{x}(\mathbf{r})\right) G_{\mathbf{r}, \mathbf{r}^{\prime}}^{ \pm}\left(E_{F}\right)\right]\left[\left(\frac{\hbar}{i} \nabla_{x}^{\prime}-e A_{x}\left(\mathbf{r}^{\prime}\right)\right) G_{\mathbf{r}^{\prime}, \mathbf{r}}^{ \pm}\left(E_{F}\right)\right]
$$

and

$$
\sigma_{x x}^{+-}=2 \frac{e^{2} \pi \hbar}{\Omega}\left(\frac{1}{2 \pi m}\right)^{2} \int d^{2} r d^{2} r^{\prime}\left[\left(\frac{\hbar}{i} \nabla_{x}-e A_{x}(\mathbf{r})\right) G_{\mathbf{r}, \mathbf{r}^{\prime}}^{-}\left(E_{F}\right)\right]\left[\left(\frac{\hbar}{i} \nabla_{x}^{\prime}-e A_{x}\left(\mathbf{r}^{\prime}\right)\right) G_{\mathbf{r}^{\prime}, \mathbf{r}}^{+}\left(E_{F}\right)\right] .
$$

Here, $G_{\mathbf{r}, \mathbf{r}^{\prime}}^{ \pm}(E)=\left\langle\mathbf{r}\left|\hat{G}^{ \pm}(E)\right| \mathbf{r}^{\prime}\right\rangle$ denotes the matrix elements of the Green functions. It is shown in appendix $\mathbb{Q}$ that both $\sigma_{x x}^{++}$and $\sigma_{x x}^{--}$vanish in the semiclassical approximation. Hence, we focus on $\sigma_{x x}^{+-}$in the remainder of this paper.

Having expressed the longitudinal conductivity in terms of Green functions, we now approximate these by their semiclassical expressions. Generally, the semiclassical Green function $G_{\mathbf{r}^{\prime}, \mathbf{r}}^{+}(E)$ is a sum over all classical trajectories of energy $E$ from $\mathbf{r}$ to $\mathbf{r}^{\prime}$. For free electrons in a magnetic field, there are an infinite number of trajectories connecting $\mathbf{r}$ to $\mathbf{r}^{\prime}$ 
if the distance between $\mathbf{r}$ and $\mathbf{r}^{\prime}$ is less than twice the cyclotron radius $R_{c}$ : Two different cyclotron orbits labeled by $S$ (for short) and $L$ (for long) which can each be traversed completely for $n=0,1,2 \ldots$ times pass through both points (cf. Fig. 1). There are no trajectories from $\mathbf{r}$ to $\mathbf{r}^{\prime}$ when $\left|\mathbf{r}-\mathbf{r}^{\prime}\right|>2 R_{c}$ and the semiclassical Green function vanishes for such arguments. An explicit calculation shown in appendix A gives

$$
G_{\mathbf{r}^{\prime}, \mathbf{r}}^{+}(E)=\frac{m}{2 i \hbar} \sum_{n=0}^{\infty} \sum_{q=S, L}\left(\frac{\omega_{c}}{\pi i \hbar E\left|\sin \left(\omega_{c} T_{n, q}\right)\right|}\right)^{1 / 2} \exp \left(-\frac{T_{n, q}}{2 \tau_{e l}}\right) \exp \left\{\frac{i}{\hbar} S_{n, q}-\frac{i \pi}{2} \eta_{n, q}\right\} .
$$

Here $T_{n, q}$ denotes the traversal time of the trajectory $(n, q)$,

$$
T_{n, q}=\frac{2 \pi n}{\omega_{c}}+t_{q}
$$

where

$$
\begin{aligned}
& t_{S}=\frac{2}{\omega_{c}} \arcsin \left(\left|\mathbf{r}-\mathbf{r}^{\prime}\right| / 2 R_{c}\right) \\
& t_{L}=\frac{2}{\omega_{c}}\left[\pi-\arcsin \left(\left|\mathbf{r}-\mathbf{r}^{\prime}\right| / 2 R_{c}\right)\right]
\end{aligned}
$$

are the times for going from $\mathbf{r}$ to $\mathbf{r}^{\prime}$ without completely traversing the cyclotron orbit $\left(\omega_{c}=\right.$ $e B / m$ is the cyclotron frequency). Each trajectory contributes with a phase factor given by its classical action

$$
S_{n, q}(E)=E T_{n, q}+\frac{m \omega_{c}}{2}\left[\frac{\left|\mathbf{r}-\mathbf{r}^{\prime}\right|^{2}}{2} \cot \left(\frac{\omega_{c} T_{n, q}}{2}\right)-\left(x y^{\prime}-x^{\prime} y\right)\right]
$$

and the Maslov indices $\eta_{n, S}=2 n, \eta_{n, L}=2 n+1$. The corresponding expression for $G_{\mathbf{r}, \mathbf{r}^{\prime}}^{-}(E)$ follows from the relation

$$
G_{\mathbf{r}, \mathbf{r}^{\prime}}^{-}(E)=\left[G_{\mathbf{r}^{\prime}, \mathbf{r}}^{+}(E)\right]^{*}
$$

One notes that both Green functions appearing in (5) are given in terms of trajectories from $\mathbf{r}$ to $\mathbf{r}^{\prime}$

Substituting these semiclassical expressions for the Green functions into Eq. (5) for the conductivity one has a double sum over classical trajectories $(n, q)$ from $\mathbf{r}$ to $\mathbf{r}^{\prime}$, 


$$
\begin{aligned}
\sigma_{x x}= & \frac{\omega_{c}}{2 \Omega E_{F}}\left(\frac{m e}{2 \pi \hbar}\right)^{2} \sum_{n_{1}=0}^{\infty} \sum_{n_{2}=0}^{\infty} \sum_{q_{1}=S, L} \sum_{q_{2}=S, L} \int d^{2} r \int d^{2} r^{\prime}\left(v_{q_{1}}\right)_{x}\left(v_{q_{2}}^{\prime}\right)_{x} \\
& \times \frac{\exp \left\{-\left(T_{n_{1}, q_{1}}+T_{n_{2}, q_{2}}\right) / 2 \tau_{e l}\right\}}{\left|\sin \left(\omega_{c} T_{n_{1}, q_{1}}\right) \sin \left(\omega_{c} T_{n_{2}, q_{2}}\right)\right|^{1 / 2}} \exp \left\{\frac{i}{\hbar}\left[S_{n_{2}, q_{2}}-S_{n_{1}, q_{1}}\right]-\frac{\pi}{2}\left[\eta_{n_{2}, q_{2}}-\eta_{n_{1}, q_{1}}\right]\right\},
\end{aligned}
$$

where we have used the relations

$$
\begin{aligned}
\frac{1}{m}\left(\frac{\hbar}{i} \nabla_{x}-e A_{x}(\mathbf{r})\right) S_{n, q} & =-\left(v_{q}\right)_{x} \\
\frac{1}{m}\left(\frac{\hbar}{i} \nabla_{x}^{\prime}-e A_{x}\left(\mathbf{r}^{\prime}\right)\right) S_{n, q} & =\left(v_{q}^{\prime}\right)_{x}
\end{aligned}
$$

Here $\left(v_{q}\right)_{x}$ and $\left(v_{q}^{\prime}\right)_{x}$ denote the $x$ components of the velocities at the points $\mathbf{r}$ and $\mathbf{r}^{\prime}$, respectively. One notes that for $q_{1}=q_{2}$, the difference in action $S_{n_{2}, q_{2}}-S_{n_{1}, q_{1}}$ is independent of both $\mathbf{r}$ and $\mathbf{r}^{\prime}$. Only these terms contribute to leading order in the semiclassical limit because the integrand oscillates rapidly when $q_{1} \neq q_{2}$. A further simplification arises because both the times $T_{n, q}$ and the difference in action depend only on $\left|\mathbf{r}-\mathbf{r}^{\prime}\right|$. It is useful to introduce center-of-mass coordinates and to change variables to the times $t_{q}$,

$$
\begin{aligned}
\int_{\Omega} d^{2} r \int_{\left|\mathbf{r}-\mathbf{r}^{\prime}\right| \leq 2 R_{c}} d^{2} r^{\prime} & =2 \pi \Omega \int_{0}^{2 R_{c}}\left|\mathbf{r}-\mathbf{r}^{\prime}\right| d\left|\mathbf{r}-\mathbf{r}^{\prime}\right| \\
& =2 \pi \Omega \omega_{c} R_{c}^{2}\left[\int_{0}^{\pi / \omega_{c}} d t_{S}\left|\sin \left(\omega_{c} t_{S}\right)\right|+\int_{\pi / \omega_{c}}^{2 \pi / \omega_{c}} d t_{L}\left|\sin \left(\omega_{c} t_{L}\right)\right|\right] .
\end{aligned}
$$

Using $\left(v_{q}\right)_{x}\left(v_{q}^{\prime}\right)_{x}=v_{x}(0) v_{x}\left(t_{q}\right)$ and combining the integrations over $t_{S}$ and $t_{L}$, one obtains

$$
\begin{aligned}
\sigma_{x x}= & \frac{e^{2} m}{2 \pi \hbar^{2}} \int_{0}^{2 \pi / \omega_{c}} d t v_{x}(0) v_{x}(t) e^{-t / 2 \tau_{e l}} \\
& \times \sum_{n_{1}, n_{2}=0}^{\infty} \exp \left\{-\frac{\pi}{\omega_{c} \tau_{e l}}\left(n_{1}+n_{2}\right)\right\} \cos \left\{2 \pi\left(\frac{E_{F}}{\hbar \omega_{c}}-\frac{1}{2}\right)\left(n_{2}-n_{1}\right)\right\} .
\end{aligned}
$$

Finally performing the sum over $n_{1}+n_{2}$ and introducing $p=\left|n_{1}-n_{2}\right|$ we find

$$
\sigma_{x x}=\frac{2 e^{2}}{h}\left(\frac{E_{F}}{2 \pi \hbar \omega_{c}}\right) C\left(v_{x}, v_{x}\right)\left[1+2 \sum_{p=1}^{\infty} \exp \left(-\frac{p \pi}{\omega_{c} \tau_{e l}}\right) \cos \left\{2 \pi p\left(\frac{E_{F}}{\hbar \omega_{c}}-\frac{1}{2}\right)\right\}\right] .
$$

Here we introduced the correlation function

$$
C\left(v_{x}, v_{x}\right)=\frac{1}{R_{c}^{2}} \int_{0}^{2 \pi / \omega_{c}} d t \int_{0}^{\infty} d t^{\prime} v_{x}(t) v_{x}\left(t+t^{\prime}\right) e^{-t^{\prime} / \tau_{e l}}
$$


This way of writing the result will be useful for comparison with the conductivity of the antidot lattice derived in the next section. For comparison with the standard result for Shubnikov-de Haas oscillations in the literature [27] one easily computes

$$
C\left(v_{x}, v_{x}\right)=\frac{\pi \omega_{c} \tau_{e l}}{1+\left(\omega_{c} \tau_{e l}\right)^{2}}
$$

Using that the density of electrons (per spin) is $n_{e}=m E_{F} / 2 \pi \hbar^{2}$ the prefactor in Eq. (16) can be rewritten in the more familiar form $\left(n_{e} e^{2} \tau_{e l} / m\right)\left[1+\left(\omega_{c} \tau_{e l}\right)^{2}\right]^{-1}$. This proves that the semiclassical approximation reproduces the low-field approximation $\left(E_{F} \gg \hbar \omega_{c}\right)$ to the quantum-mechanical result (within the Born approximation) 27. We note that the classical Drude conductivity arises from the diagonal terms in the double sum over trajectories in Eq. (11). The Shubnikov-de Haas oscillations arise from non-diagonal terms corresponding to different numbers $n_{1}$ and $n_{2}$ of complete revolutions around the same cyclotron orbit. In the next section we will find a closely analogous structure of the terms contributing to the conductivity of antidot lattices.

\section{QUANTUM TRANSPORT IN ANTIDOT LATTICES}

\section{A. Longitudinal conductivity}

In the following we consider the longitudinal conductivity of macroscopic antidot lattices. We start from the Kubo formula (1) with the Hamiltonian $\hat{H}=(1 / 2 m)(\hat{\mathbf{p}}-e \mathbf{A})^{2}+V(\hat{\mathbf{r}})$ where $V(\hat{\mathbf{r}})$ is the antidot potential. A semiclassical approximation to the Green function

$G_{\mathbf{r}^{\prime}, \mathbf{r}}^{+}(E)$ for general potentials $V(\mathbf{r})$ is obtained by making consistent use of stationary-phase integration both for the path integral for the propagator and for the Fourier transform to the energy domain. One finds that the Green-function matrix elements are approximated by sums over the classical trajectories $\gamma\left(\mathbf{r}, \mathbf{r}^{\prime}\right)$ of energy $E$ going from $\mathbf{r}$ to $\mathbf{r}^{\prime}$ [24,

$$
G_{\mathbf{r}^{\prime}, \mathbf{r}}^{+}(E)=\frac{1}{(2 \pi)^{1 / 2}}\left(\frac{1}{i \hbar}\right)^{3 / 2} \sum_{\gamma\left(\mathbf{r}, \mathbf{r}^{\prime}\right)} D_{\gamma}\left(\mathbf{r}, \mathbf{r}^{\prime}\right) \exp \left\{\frac{i}{\hbar} S_{\gamma}\left(\mathbf{r}, \mathbf{r}^{\prime} ; E\right)-i \frac{\pi}{2} \eta_{\gamma}\right\}
$$


The phase of the Green function is determined by the classical action $S_{\gamma}\left(\mathbf{r}, \mathbf{r}^{\prime} ; E\right)=\int_{\gamma} d \mathbf{r} \mathbf{p}$ of the trajectory $\gamma$ ( $\mathbf{p}$ is the canonical momentum and the integral is taken along the trajectory) and by the Maslov index $\eta_{\gamma}$, which counts the number of conjugate points along the trajectory. Each trajectory contributes with an amplitude $D_{\gamma}$ which is determined by disorder and the classical phase-space density,

$$
D_{\gamma}\left(\mathbf{r}, \mathbf{r}^{\prime}\right)=\exp \left(-\frac{T_{\gamma}}{2 \tau_{e l}}\right)\left|\begin{array}{cc}
\frac{\partial^{2} S_{\gamma}}{\partial r_{i}^{\prime} \partial r_{j}} & \frac{\partial^{2} S_{\gamma}}{\partial E \partial r_{j}} \\
\frac{\partial^{2} S_{\gamma}}{\partial r_{i}^{\prime} \partial E} & \frac{\partial^{2} S_{\gamma}}{\partial^{2} E}
\end{array}\right|^{1 / 2}
$$

Here the traversal time of the trajectory is denoted by $T_{\gamma}$. The corresponding expression for $G_{\mathbf{r}, \mathbf{r}^{\prime}}^{-}(E)$ follows from $G_{\mathbf{r}, \mathbf{r}^{\prime}}^{-}(E)=\left[G_{\mathbf{r}^{\prime}, \mathbf{r}}^{+}(E)\right]^{*}$. Importantly, both Green functions appearing in (5) are given in terms of trajectories running from $\mathbf{r}$ to $\mathbf{r}^{\prime}$. The main difference between the semiclassical expressions for $G_{\mathbf{r}^{\prime}, \mathbf{r}}^{+}(E)$ and $G_{\mathbf{r}, \mathbf{r}^{\prime}}^{-}(E)$ is that the actions enter in the exponent with opposite signs.

Inserting the semiclassical expressions for the Green functions into Eq. (5) one finds that $\sigma_{x x}$ is given by a double sum over classical trajectories from $\mathbf{r}$ to $\mathbf{r}^{\prime}$. In the simplest approximation one retains only the diagonal terms in this double sum for which the rapidlyoscillating phase factors drop out,

$$
\sigma_{x x} \simeq 2 \frac{e^{2} \pi \hbar}{\Omega}\left(\frac{1}{2 \pi \hbar}\right)^{3} \int d^{2} r d^{2} r^{\prime} \sum_{\gamma\left(\mathbf{r}, \mathbf{r}^{\prime}\right)} v_{x} v_{x}^{\prime} D_{\gamma}^{2}\left(\mathbf{r}, \mathbf{r}^{\prime}\right)
$$

Here we used the elementary relations

$$
\begin{aligned}
& \frac{1}{m}\left(\frac{\hbar}{i} \nabla_{x}-e A_{x}(\mathbf{r})\right) S_{\gamma}\left(\mathbf{r}, \mathbf{r}^{\prime} ; E_{F}\right)=-v_{x} \\
& \frac{1}{m}\left(\frac{\hbar}{i} \nabla_{x}^{\prime}-e A_{x}\left(\mathbf{r}^{\prime}\right)\right) S_{\gamma}\left(\mathbf{r}, \mathbf{r}^{\prime} ; E_{F}\right)=v_{x}^{\prime}
\end{aligned}
$$

where $v_{x}$ and $v_{x}^{\prime}$ denote the $x$ components of the initial and final velocities, respectively. This contribution gives the classical conductivity. To see this, we multiply the right-hand side of Eq. (21) by $1=\int d E \delta\left(E-E_{F}\right)$ and introduce as new integration variables the initial position $\mathbf{r}$, the initial momentum $\mathbf{p}$, and the traversal time $t$ of the trajectory $\gamma$. The corresponding Jacobian is given by 


$$
d^{2} r d^{2} p d t=d^{2} r d^{2} r^{\prime} d E \sum_{\gamma\left(\mathbf{r}, \mathbf{r}^{\prime}\right)}\left|\begin{array}{cc}
\frac{\partial p_{i}}{\partial r_{j}^{\prime}} & \frac{\partial p_{i}}{\partial E} \\
\frac{\partial t}{\partial r_{j}^{\prime}} & \frac{\partial t}{\partial E}
\end{array}\right|
$$

The sum over $\gamma$ appears because initial conditions $(\mathbf{r}, \mathbf{p})$ specify a classical trajectory uniquely while boundary conditions $\left(\mathbf{r}, \mathbf{r}^{\prime}\right)$ can be satisfied by more than one trajectory. One notes that the Jacobian (24) involves precisely the same determinant which also appears in the amplitude factors $D_{\gamma}\left(\mathbf{r}, \mathbf{r}^{\prime}\right)$ in the Green functions (19). Hence, one finds for the conductivity

$$
\sigma_{x x} \simeq \frac{e^{2}}{h^{2} \Omega} \int d^{2} r d^{2} p d t v_{x}(0) v_{x}(t) e^{-t / \tau_{e l}} \delta\left(E_{F}-H(\mathbf{p}, \mathbf{r})\right)
$$

where $H(\mathbf{p}, \mathbf{r})$ denotes the classical Hamiltonian. Introducing the classical phase-space averages

$$
\langle f(\mathbf{p}, \mathbf{r})\rangle_{\Gamma}=\frac{1}{h^{2} \Omega N(0)} \int d^{2} r d^{2} p f(\mathbf{p}, \mathbf{r}) \delta\left(E_{F}-H(\mathbf{p}, \mathbf{r})\right)
$$

$\left(N(0)=m / 2 \pi \hbar^{2}\right.$ is the density of states at the Fermi energy in two dimensions) the conductivity can be rewritten in the familiar form

$$
\sigma_{x x} \simeq e^{2} N(0) \int_{0}^{\infty} d t\left\langle v_{x}(0) v_{x}(t)\right\rangle_{\Gamma} e^{-t / \tau_{e l}}
$$

This is precisely the classical Kubo formula. Hence, the diagonal approximation to the double sum over classical trajectories in Eq. (5) is equivalent to the classical approximation. At first sight, it may appear inconsistent that the classical limit (27) still - via the density of states $N(0)$ - involves Planck's constant $\hbar$. However, this is simply a consequence of the fact that we use the Fermi-Dirac distribution instead of the Boltzmann distribution appropriate for a complete classical limit.

As shown by Fleischmann et al. 25] the classical Kubo formula (27) explains the peaks in the magnetoresistance when the cyclotron radius and the lattice spacing of the antidots are commensurate. In this paper, we are mainly interested in the quantum corrections $\delta \sigma_{x x}$ to the classical conductivity. Clearly, they must arise from the nondiagonal terms in the double sum in (5), 


$$
\begin{aligned}
\delta \sigma_{x x}=\frac{e^{2}}{h^{2} \Omega} & \int d^{2} r d^{2} r^{\prime} \sum_{\gamma_{1}\left(\mathbf{r}, \mathbf{r}^{\prime}\right) \neq \gamma_{2}\left(\mathbf{r}, \mathbf{r}^{\prime}\right)}\left(v_{1}\right)_{x}\left(v_{2}^{\prime}\right)_{x} D_{\gamma_{1}}\left(\mathbf{r}, \mathbf{r}^{\prime}\right) D_{\gamma_{2}}\left(\mathbf{r}, \mathbf{r}^{\prime}\right) \\
& \times \exp \left\{\frac{i}{\hbar}\left[S_{\gamma_{2}}\left(\mathbf{r}, \mathbf{r}^{\prime} ; E_{F}\right)-S_{\gamma_{1}}\left(\mathbf{r}, \mathbf{r}^{\prime} ; E_{F}\right)\right]-\frac{i \pi}{2}\left[\eta_{\gamma_{2}}-\eta_{\gamma_{1}}\right]\right\} .
\end{aligned}
$$

In these terms, the phase factors do not cancel and hence the integrand involves rapidlyoscillating factors. Within the semiclassical approach for completely chaotic systems, it is natural to evaluate the spatial integrals by the stationary-phase method. This procedure becomes exact in the semiclassical limit $\hbar \rightarrow 0$. Considering the contribution arising from trajectories $\gamma_{1}\left(\mathbf{r}, \mathbf{r}^{\prime}\right)$ and $\gamma_{2}\left(\mathbf{r}, \mathbf{r}^{\prime}\right)$, the stationary-phase conditions for the $\mathbf{r}$ and $\mathbf{r}^{\prime}$ integrations are

$$
\begin{gathered}
\nabla\left[S_{\gamma_{2}}\left(\mathbf{r}, \mathbf{r}^{\prime} ; E_{F}\right)-S_{\gamma_{1}}\left(\mathbf{r}, \mathbf{r}^{\prime} ; E_{F}\right)\right]=\mathbf{p}_{1}-\mathbf{p}_{2}=0 \\
\nabla^{\prime}\left[S_{\gamma_{2}}\left(\mathbf{r}, \mathbf{r}^{\prime} ; E_{F}\right)-S_{\gamma_{1}}\left(\mathbf{r}, \mathbf{r}^{\prime} ; E_{F}\right)\right]=\mathbf{p}_{2}^{\prime}-\mathbf{p}_{1}^{\prime}=0 .
\end{gathered}
$$

Here, $\mathbf{p}_{i}$ and $\mathbf{p}_{i}^{\prime}$ denote the initial and final (canonical) momenta of trajectory $\gamma_{i}$. Since the vector potential cancels from the stationary-phase conditions, one finds that the integrand becomes stationary when initial and final velocities of $\gamma_{1}$ and $\gamma_{2}$ are identical. Since also the initial and final positions of the two trajectories must each coincide one might at first think that this implies $\gamma_{1}=\gamma_{2}$ which would just be the classical contribution discussed above. However, there is an additional possibility to satisfy the stationary-phase conditions (29, 30) when the two trajectories are part of a classical periodic orbit. Then $\gamma_{1}$ and $\gamma_{2}$ can differ by an integer number $p$ of traversals of the orbit as illustrated in Fig. 2. In fact, for each (primitive) periodic orbit $\gamma$ there is an infinite set of pairs of trajectories $\gamma_{1}$ and $\gamma_{2}$ which satisfy the stationary-phase condition (29, 30). The pairs are labeled by $p$ which was defined above and by $n$ which counts the number of times the shorter trajectory from $\mathbf{r}$ to $\mathbf{r}^{\prime}$ returns to its starting point $\mathbf{r}$. It is these quantum corrections to the conductivity which we focus on in the following.

The evaluation of the stationary-phase integrations over $\mathbf{r}$ and $\mathbf{r}^{\prime}$, while different in detail, is closely analogous to performing the final trace in the derivation of Gutzwiller's 
trace formula [24]. First we note that the saddle-point action is just given by the action $S_{\gamma}\left(E_{F}\right)$ of the (primitive) periodic orbit,

$$
S_{\gamma_{2}}\left(\mathbf{r}, \mathbf{r}^{\prime} ; E_{F}\right)-S_{\gamma_{1}}\left(\mathbf{r}, \mathbf{r}^{\prime} ; E_{F}\right)=p S_{\gamma}\left(E_{F}\right)
$$

To evaluate the integral over the quadratic fluctuations around the stationary-phase point it is useful to introduce a new set of orthogonal coordinates $\mathbf{r}=(z, y)$ where $z$ measures the arclength along the periodic orbit $\gamma$ and $y$ measures the transverse deviations from the orbit (cf. Ref. [24]). The Jacobian of this transformation is unity, $d^{2} r d^{2} r^{\prime}=d z d y d z^{\prime} d y^{\prime}$. While the integrals over $y$ and $y^{\prime}$ can be done by the stationary-phase method, the integrals along the periodic orbit must be performed exactly because the second variation of the action vanishes in this direction. Introducing the matrix $F$ of second derivatives of $S_{21}=S_{\gamma_{2}}-S_{\gamma_{1}}$ with respect to $y$ and $y^{\prime}$,

$$
F=\left[\begin{array}{cc}
\partial_{y}^{2} S_{21} & \partial_{y} \partial_{y^{\prime}} S_{21} \\
\partial_{y^{\prime}} \partial_{y} S_{21} & \partial_{y^{\prime}}^{2} S_{21}
\end{array}\right]
$$

(here we used the shorthand notation $\partial_{y}=\partial / \partial y$ ) and the vector $\mathbf{y}^{T}=\left[y, y^{\prime}\right]$ of transverse deviations from the periodic orbit, one finds for the fluctuation integral

$$
\int d y d y^{\prime} \exp \left\{\frac{i}{2 \hbar} \mathbf{y}^{T} F \mathbf{y}\right\}=\frac{2 \pi i \hbar}{\sqrt{\operatorname{det} F}}
$$

A straight-forward but tedious calculation presented in appendix B shows that this fluctuation amplitude combined with the semiclassical amplitudes of the Green functions can be expressed in terms of the monodromy matrix $M_{\gamma}$ of the periodic orbit alone,

$$
\frac{D_{\gamma_{1}}\left(\mathbf{r}, \mathbf{r}^{\prime}\right) D_{\gamma_{2}}\left(\mathbf{r}, \mathbf{r}^{\prime}\right)}{\sqrt{|\operatorname{det} F|}}=\frac{\exp \left\{-\left(T_{\gamma_{1}}+T_{\gamma_{2}}\right) / 2 \tau_{e l}\right\}}{|\mathbf{v}(z)|\left|\mathbf{v}\left(z^{\prime}\right)\right|}\left|\operatorname{Tr} M_{\gamma}^{p}-2\right|^{-1 / 2} .
$$

Finally, we simplify the integrations along the periodic orbit over $z$ and $z^{\prime}$. Since the integrations over $\mathbf{r}$ and $\mathbf{r}^{\prime}$ are over the volume of the system, the integrations over $z$ and $z^{\prime}$ extend over a full period around the primitive periodic orbit. Furthermore, since $\mathbf{r}$ is the starting and $\mathbf{r}^{\prime}$ the end point of the trajectories, the limits of integration are $0 \leq z \leq L_{\gamma}$ 
and $z \leq z^{\prime} \leq z^{\prime}+L_{\gamma}$, where $L_{\gamma}$ is the length of the (primitive) periodic orbit. Hence, the $z, z^{\prime}$ integrations become

$$
\begin{gathered}
\sum_{n=0}^{\infty} \int_{0}^{L_{\gamma}} d z \int_{z}^{z+L_{\gamma}} d z^{\prime} \frac{v_{x}(z) v_{x}\left(z^{\prime}\right)}{|\mathbf{v}(z)|\left|\mathbf{v}\left(z^{\prime}\right)\right|} \exp \left\{-\left(T_{\gamma_{1}}+T_{\gamma_{2}}\right) / 2 \tau_{e l}\right\} \\
\quad=\exp \left(-p T_{\gamma} / 2 \tau_{e l}\right) \sum_{n=0}^{\infty} \int_{0}^{T_{\gamma}} d t \int_{0}^{T_{\gamma}} d t^{\prime} v_{x}(t) v_{x}\left(t+t^{\prime}\right) \exp \left\{-\left(n T_{\gamma}+t^{\prime}\right) / \tau_{e l}\right\} \\
=\exp \left(-p T_{\gamma} / 2 \tau_{e l}\right) \int_{0}^{T_{\gamma}} d t \int_{0}^{\infty} d t^{\prime} v_{x}(t) v_{x}\left(t+t^{\prime}\right) \exp \left(-t^{\prime} / \tau_{e l}\right) .
\end{gathered}
$$

Here, $T_{\gamma}=L_{\gamma} / v_{F}$ denotes the period of the primitive periodic orbit. Due to the translational symmetry of the antidot lattice each periodic orbit appears $\Omega / a^{2}$ times $(a$ is the lattice spacing). Including only a single representative of each class of periodic orbits in the sum over $\gamma$, we find the final result

$$
\delta \sigma_{x x}=\frac{2 e^{2}}{h} \frac{R_{c}^{2}}{a^{2}} \sum_{\gamma} \sum_{p=1}^{\infty} \exp \left(-p T_{\gamma} / 2 \tau_{e l}\right) C_{\gamma}\left(v_{x}, v_{x}\right) \frac{\cos \left\{p S_{\gamma}\left(E_{F}\right) / \hbar-\pi p \alpha_{\gamma} / 2\right\}}{\left|\operatorname{Tr} M_{\gamma}^{p}-2\right|^{1 / 2}},
$$

where the sum over $\gamma$ is over all primitive periodic orbits and $p$ counts the repeated traversals. We also introduced the correlation function

$$
C_{\gamma}\left(v_{x}, v_{x}\right)=\frac{1}{R_{c}^{2}} \int_{0}^{T_{\gamma}} d t \int_{0}^{\infty} d t^{\prime} v_{x}(t) v_{x}\left(t+t^{\prime}\right) \exp \left(-t^{\prime} / \tau_{e l}\right)
$$

Some periodic orbits of antidot lattices turn out to be quite close to circular. For these it can be a useful approximation to replace the present correlation function by its analog (17) for Shubnikov-de Haas oscillations. By collecting all phases one convinces oneself that the Maslov index $\alpha_{\gamma}$ appearing in Eq. (36) is the same that enters Gutzwiller's trace formula [24]. Hence, periodic-orbit contributions to the conductivity and to the density of states have the same phase which is physically reasonable. The result (36) for the periodic-orbit corrections to the conductivity of antidot lattices should be compared to the result (16) for Shubnikov-de Haas oscillations. The principal difference is that the stability of the periodic orbit expressed in terms of the monodromy matrix enters in the case of chaotic dynamics. Furthermore, one notes that the quantum oscillations for the antidot lattices are of a higher power in Planck's constant $\hbar$ and hence smaller in the semiclassical limit than the Shubnikov-de Haas oscillations. 
At finite temperatures the Kubo formula takes on the form

$$
\sigma_{x x}=-\frac{e^{2} \pi \hbar}{\Omega} \int_{0}^{\infty} d E \frac{\partial f_{\mu}(E)}{\partial E} \operatorname{Tr}\left\{\hat{v}_{x} \delta_{\Gamma}(E-\hat{H}) \hat{v}_{x} \delta_{\Gamma}(E-\hat{H})\right\}
$$

where $\mu$ denotes the chemical potential and $f_{\mu}(E)$ is the Fermi-Dirac distribution $f_{\mu}(E)=$ $[1+\exp (\beta(E-\mu))]^{-1}\left(\beta=1 / k_{B} T\right.$ is the inverse temperature). To compute the temperature dependence of the periodic-orbit contribution to the conductivity one notes that the dominant energy dependence (to leading order in $\hbar$ ) is in the phase factor. Hence, we need to compute the integral

$$
I(T)=-\int_{0}^{\infty} d E \frac{\partial f_{\mu}(E)}{\partial E} \cos \left\{\frac{p}{\hbar} S_{\gamma}(E)-\frac{\pi}{2} p \alpha_{\gamma}\right\}
$$

Since the derivative of the Fermi function is strongly peaked at the Fermi energy, we extend the lower limit of integration to $-\infty$ and expand the action to first order around the Fermi energy,

$$
I(T)=\operatorname{Re} \frac{\partial}{\partial \mu} \exp \left\{\frac{i}{\hbar} p S_{\gamma}(\mu)-\frac{i \pi}{2} p \alpha_{\gamma}\right\} \int_{-\infty}^{\infty} d E f_{\mu}(E) \exp \left\{\frac{i}{\hbar} p T_{\gamma}(E-\mu)\right\}
$$

Here we used that $d S_{\gamma} / d E=T_{\gamma}$. This integral can be evaluated by contour integration. The Fermi-Dirac distribution has poles at $E=\mu+i(2 n+1) \pi / \beta$ with residues $-1 / \beta$. One finds

$$
I(T)=\cos \left\{\frac{p}{\hbar} S_{\gamma}(\mu)-\frac{\pi}{2} p \alpha_{\gamma}\right\} \frac{\left(\pi p T_{\gamma} / \hbar \beta\right)}{\sinh \left(\pi p T_{\gamma} / \hbar \beta\right)}
$$

Thus we find for the periodic-orbit contribution to the conductivity at finite temperature

$$
\begin{aligned}
& \delta \sigma_{x x}=\frac{2 e^{2}}{h} \frac{R_{c}^{2}}{a^{2}} \sum_{\gamma} \sum_{p=1}^{\infty} \exp \left(-p T_{\gamma} / 2 \tau_{e l}\right) C_{\gamma}\left(v_{x}, v_{x}\right) \\
& \times \frac{\left(\pi p T_{\gamma} / \hbar \beta\right)}{\sinh \left(\pi p T_{\gamma} / \hbar \beta\right)} \frac{\cos \left\{p S_{\gamma}\left(E_{F}\right) / \hbar-\pi p \alpha_{\gamma} / 2\right\}}{\left|\operatorname{Tr} M_{\gamma}^{p}-2\right|^{1 / 2}} .
\end{aligned}
$$

The contribution from each periodic orbit decreases exponentially with temperature on the scale of $\hbar / T_{\gamma}$. In principle, there is an addional temperature dependence due to the variation of the inelastic scattering length with temperature. However, this temperature dependence 
is slow compared to that arising from thermal smearing. For this reason, Eq. (42) does indeed describe the dominant temperature dependence of the effect.

The Zeeman coupling of the electronic spin to the magnetic field is readily incorporated into our results. It effectively leads to separate Fermi energies $E_{F} \pm \mu_{B} B$ for the two spin components (here $\mu_{B}=e \hbar / 2 m_{e}$ is the Bohr magneton with $m_{e}$ the bare electron mass). Expanding the action to linear order in the Zeeman energy one finds that it leads to beating of the oscillation amplitude,

$$
\begin{aligned}
\delta \sigma_{x x}=\frac{2 e^{2}}{h} & \frac{R_{c}^{2}}{a^{2}} \sum_{\gamma} \sum_{p=1}^{\infty} \exp \left(-p T_{\gamma} / 2 \tau_{e l}\right) C_{\gamma}\left(v_{x}, v_{x}\right) \\
& \times \cos \left(p T_{\gamma} \mu_{B} B / \hbar\right) \frac{\left(\pi p T_{\gamma} / \hbar \beta\right)}{\sinh \left(\pi p T_{\gamma} / \hbar \beta\right)} \frac{\cos \left\{p S_{\gamma}\left(E_{F}\right) / \hbar-\pi p \alpha_{\gamma} / 2\right\}}{\left|\operatorname{Tr} M_{\gamma}^{p}-2\right|^{1 / 2}} .
\end{aligned}
$$

The corresponding magnetic-field scale is $\hbar / \mu_{B} T_{\gamma}$.

\section{B. Hall conductivity}

The calculation for the Hall conductivity $\sigma_{x y}$ is closely analogous to that for the longitudinal conductivity described in the previous section. The Hall conductivity can be expressed in terms of two contributions 36

$$
\sigma_{x y}=\sigma_{x y}^{I}+\sigma_{x y}^{I I}
$$

where

$$
\sigma_{x y}^{I}=\frac{i e^{2} \hbar}{2 \Omega} \operatorname{Tr}\left\{\hat{v}_{x} \hat{G}^{+}\left(E_{F}\right) \hat{v}_{y} \delta_{\Gamma}\left(E_{F}-H\right)-\hat{v}_{x} \delta_{\Gamma}\left(E_{F}-H\right) \hat{v}_{y} \hat{G}^{-}\left(E_{F}\right)\right\}
$$

and

$$
\sigma_{x y}^{I I}=-\frac{e}{\Omega} \int_{0}^{E_{F}} d E \frac{\partial}{\partial B} \rho(E)
$$

Here, $\rho(E)$ denotes the density of states. First, we evaluate the contribution $\sigma_{x y}^{I I}$ using Gutzwiller's trace formula which gives a semiclassical approximation to the density of states $\rho(E)$. The Weyl contribution to the density of states is independent of the magnetic field. 
Thus, we only need the oscillatory contributions $\delta \rho(E)$ to the density of states which can be expressed in terms of a sum over the periodic orbits $\gamma$ of the system,

$$
\delta \rho(E)=\frac{\Omega}{a^{2}} \frac{1}{\pi \hbar} \sum_{\gamma} \sum_{p=1}^{\infty} \frac{T_{\gamma} \exp \left(-p T_{\gamma} / 2 \tau_{e l}\right)}{\left|\operatorname{Tr} M_{\gamma}^{p}-2\right|^{1 / 2}} \cos \left\{\frac{1}{\hbar} p S_{\gamma}(E)-\frac{\pi}{2} p \alpha_{\gamma}\right\} .
$$

Noting that to leading order in Planck's constant $\hbar$ we only need to keep the magnetic-field and energy dependence in the oscillatory factor, one readily finds

$$
\delta \sigma_{x y}^{I I}=-\frac{2 e^{2}}{h} \sum_{\gamma} \sum_{p=1}^{\infty} \exp \left(-p T_{\gamma} / 2 \tau_{e l}\right)\left(\frac{1}{a^{2}} \frac{\partial S_{\gamma}\left(E_{F}\right)}{\partial B}\right) \frac{\cos \left\{p S_{\gamma}\left(E_{F}\right) / \hbar-\pi p \alpha_{\gamma} / 2\right\}}{\left|\operatorname{Tr} M_{\gamma}^{p}-2\right|^{1 / 2}} .
$$

We now proceed to evaluate the first contribution $\sigma_{x y}^{I}$. The evaluation of this term is completely analogous to that for $\sigma_{x x}^{+-}$. We do not consider the classical Hall conductivity and focus on the periodic-orbit contribution right away. Again, we only need to consider contributions from products of advanced and retarded Green functions. By analogy with the evaluation of $\sigma_{x y}^{+-}$we can immediately write down the result of the calculation for the Hall conductivity,

$$
\delta \sigma_{x y}^{I}=\frac{2 e^{2}}{h} \frac{R_{c}^{2}}{a^{2}} \sum_{\gamma} \sum_{p=1}^{\infty} \exp \left(-p T_{\gamma} / 2 \tau_{e l}\right) C_{\gamma}\left(v_{x}, v_{y}\right) \frac{\cos \left\{p S_{\gamma}\left(E_{F}\right) / \hbar-p \pi \alpha_{\gamma} / 2\right\}}{\left|\operatorname{Tr} M_{\gamma}^{p}-2\right|^{1 / 2}}
$$

where the velocity correlations are given by

$$
C_{\gamma}\left(v_{x}, v_{y}\right)=\frac{1}{R_{c}^{2}} \int_{0}^{T_{\gamma}} d t \int_{0}^{\infty} d t^{\prime} v_{x}(t) v_{y}\left(t+t^{\prime}\right) e^{-t^{\prime} / \tau_{e l}} .
$$

The Maslov index $\alpha_{\gamma}$ is again the same that also appears in Gutzwiller's trace formula. Finite temperature and the Zeeman splitting lead to the same additional factors which also appeared in the treatment of the longitudinal conductivity in the preceding section. For an approximately circular periodic orbit of radius $R$ traversed at constant angular velocity $\omega$ one finds

$$
\delta \sigma_{x y}^{\mathrm{cir}} \approx \frac{2 e^{2}}{h} \frac{\pi R^{2}}{a^{2}} \frac{1}{1+\left(\omega \tau_{e l}\right)^{2}} \exp \left(-p T_{\gamma} / 2 \tau_{e l}\right) \frac{\cos \left[p S_{\gamma}\left(E_{F}\right) / \hbar-p \pi \alpha_{\gamma} / 2\right]}{\left|\operatorname{Tr} M_{\gamma}^{p}-2\right|^{1 / 2}} .
$$

The same disorder-dependent prefactor appears in the theory of Shubnikov-de Haas oscillations [27]. Note that the amplitude of a circular orbit in the Hall conductivity is smaller by a factor $1 / \omega \tau_{e l}$ than that in the longitudinal conductivity. 


\section{Comparison with experiment}

Weiss et al. [5, 37] measured both the longitudinal conductivity and the Hall conductivity of macroscopic antidot lattices as function of magnetic field. The antidot lattices were patterned from high-mobility GaAs-AlGaAs heterostructures with elastic and inelastic mean free paths of order $5 \mu \mathrm{m}$. For the main sample discussed below the lattice spacing was $a=200 \mathrm{~nm}$ and the antidot diameter $d=100 \mathrm{~nm}$. The Fermi wavelength was of order $\lambda_{F}=50 \mathrm{~nm}$.

At higher temperatures (typically $T=4.7 \mathrm{~K}$ ) Weiss et al. [3] found a number of peaks in the longitudinal resistivity at magnetic fields where the classical cyclotron radius is commensurate with the lattice spacing of the antidot array. This phenomenon has been discussed theoretically by Fleischmann et al. 25]. Here we are concerned with the additional quantum oscillations in both longitudinal and Hall conductivities superimposed on the classical conductivity. These were observed by Weiss et al. [5] at subkelvin temperatures. The experimental results for the main sample investigated in Ref. [5] are shown in Fig. 3. To focus on the quantum oscillations, we have plotted $\delta \sigma=\sigma(T=0.4 \mathrm{~K})-\sigma(T=4.7 \mathrm{~K})$. In the following we compare our theoretical results to the experimental data for this particular sample. The quantum oscillations have a period of the order of one flux quantum per unit cell and the oscillations in $\sigma_{x x}$ and $\sigma_{x y}$ are in phase. One reads off from Fig. 3 that the amplitude is roughly $\delta \sigma_{x x} \approx \delta \sigma_{x y} \approx 0.015(\mathrm{k} \Omega)^{-1}$.

A detailed comparison between theory and experiment is complicated by a number of factors. Corrections to the semiclassical results may be relevant because the Fermi wavelength is not much smaller than the antidot radius. In addition, our approximations are strictly valid only for completely chaotic systems. By contrast, the experimental system exhibits mixed dynamics in part of the magnetic-field range over which the quantum oscillations have been observed. One expects that this does not affect the phase of the oscillations strongly but does have an influence on their amplitude. A detailed comparison of theory and experiment also requires use of a model for the antidot potential which is not known a 
priori. Perhaps the most serious shortcoming is our treatment of disorder which is sufficient to prove that there is a rigorous connection between quantum corrections to the conductivity and classical periodic orbits but which is not fully adequate to describe the smooth disorder potentials typical of semiconductor heterostructures. For want of a better treatment we have considered the elastic mean free path as a fit parameter, keeping it merely within reasonable bounds.

We model the antidot potential by [25]

$$
V(\mathbf{r})=V_{0}[\cos (\pi x / a) \cos (\pi y / a)]^{\beta}
$$

Here, $V_{0}$ controls the diameter of the antidots at the Fermi energy and $\beta$ fixes the steepness of the antidot potential. Hence, all parameters but $\beta$ are fixed directly by experiment. Following Ref. [5] we choose $\beta=2$. In Ref. [5] this choice was motivated by the phase of the quantum oscillations. Using the approach of Fleischmann et al. 25] we have also computed numerically the classical resistivity for this potential. Experimentally one finds a single peak located at $R_{c}=a / 2$. As shown in Fig. 4 , the model potential with $\beta=2$ also leads to a single dominant peak at the same magnetic-field value in the classical resistivity. This lends additional support to this choice of $\beta$. Other choices such as $\beta=4$ lead to considerable additional structure in the resistivity [25].

To study the nature of the classical dynamics for this choice of model potential we have generated Poincare surfaces of section. For $\beta=2$ and magnetic fields such that $R_{c}=a / 2$ one finds that phase space is entirely chaotic (within our resolution). This is shown in Fig. 5(a). The classical dynamics becomes more (less) chaotic as one decreases (increases) the magnetic field from $R_{c}=a / 2$. In particular, islands of regular motion appear for larger magnetic fields. Our results for completely chaotic systems should be applicable for magnetic fields with $R_{c}>a / 2$. However, as already mentioned above, one might expect a somewhat reduced level of accuracy because the trajectories are close to stability. The classical dynamics also becomes less chaotic as one increases the steepness of the antidot potential. This is shown in Fig. 5(b) for $\beta=4$ and $R_{c}=a / 2$, where one finds a large regular 
island in the Poincare section.

Our central result Eq. (36) expresses the quantum oscillations in the conductivity in terms of the classical periodic orbits of the antidot lattice. Since long orbits are suppressed due to elastic and inelastic scattering, stability amplitude, and finite temperature, we focus on the contributions from the shortest periodic orbits labeled by (a), (b), and (c) in Fig. 6 . It turns out that the action of orbit (a) changes only slowly with magnetic field giving rise to oscillations with large period. These are not resolved experimentally because of the small magnetic-field range. Note that while orbit (b) has fourfold symmetry, orbit (c) has only twofold symmetry so that the latter contributes twice. As already pointed out by Weiss et al. [5] these orbits explain the phase of the quantum oscillations. Both orbits have nearly the same action and one finds that the predicted minima from Eq. (36) indicated by arrows in Fig. 3 agree well with the observed minima in the conductivity. Furthermore, our results predict in agreement with experiment that longitudinal and Hall conductivity are in phase.

The amplitude of each periodic orbit $\gamma$ in Eq. (36) depends on the traversal time $T_{\gamma}$, the velocity correlations $C_{\gamma}\left(v_{x}, v_{x}\right)$, and the stability factor $\left|\operatorname{Tr} M_{\gamma}-2\right|^{-1 / 2}$. Our numerical results for these quantities at the classical peak $R_{c}=a / 2$ and for $\omega_{c} \tau_{e l}=2$ are collected in table I. Orbit (c) in fact consists of three periodic orbits in close vicinity of each other. In view of the Fermi wavelength $\lambda_{F}$ we do not believe that it is justified to include all three orbits separately. Instead we only include one of the three with typical stability. Presumably this tends to underestimate the amplitude and hence a more accurate treatment would lead to closer agreement between theory and experiment: Using the numbers of table I we find $\delta \sigma_{x x} \approx 0.008(\mathrm{k} \Omega)^{-1}$ from Eqs. (36) and $\delta \sigma_{x y} \approx 0.004(\mathrm{k} \Omega)^{-1}$ from Eq. (49) 38. These theoretical estimates are smaller by factors of two and four, respectively, than the experimental results. We believe that this order-of-magnitude agreement is reasonable given the theoretical uncertainties mentioned at the beginning of this section.

Our results predict a beat period due to the Zeeman splitting which is rather large compared to the magnetic-field range over which the quantum oscillations were observed. While the effects of the Zeeman splitting were thus unobservable, the predicted temperature 
dependence is consistent with experimental results. The amplitude of the quantum oscillations is expected to decrease exponentially with temperature on the scale $\hbar / \pi T_{\gamma}$. Hence, one predicts a characteristic temperature of $0.7 \mathrm{~K}$. This is consistent with experiment where quantum oscillations were observed at $0.4 \mathrm{~K}$ but not at $4.7 \mathrm{~K}$ [3,5].

In the introduction we remarked that the periodicity of the quantum oscillations varies between $1 / B$ periodicity and $B$ periodicity depending on the experimental sample 5 . To understand this observation, consider the magnetic-field range where the cyclotron radius is of the order of half the lattice spacing so that electrons can circle around a single antidot. If the antidot diameter is small the relevant periodic orbits are only weakly perturbed by the presence of the antidot potential. Hence, one expects $1 / B$ periodicity familiar from the Shubnikov-de Haas oscillations in unpatterned samples. On the other hand, if the antidot diameter is larger, the periodic orbits are modified by the antidot potential. In particular, they will be inhibited in contracting with increasing magnetic field. Hence, with increasing antidot diameter one crosses over to a situation where the enclosed area of the periodic orbits is approximately constant with magnetic field. In this limit the change of the classical action with magnetic field is merely due to the Aharonov-Bohm flux and hence one finds $B$ periodicity.

\section{DISCUSSION AND SUMMARY}

Motivated by a recent experiment by Weiss et al. [5] we have studied the quantum transport in large antidot lattices. We have evaluated the Kubo formula for the longitudinal and the Hall conductivity semiclassically for systems whose classical dynamics is chaotic. While the leading contribution of the semiclassical expansion is just the classical Kubo formula as expected, we have identified quantum corrections due to the classical periodic orbits of the system. The contribution from each periodic orbit oscillates as function of the Fermi energy and the magnetic field with the phase given by the classical action of the orbit. The amplitude is determined by the stability and the velocity correlations of the orbit. 
Furthermore, we find that the periodic-orbit contributions to the conductivity decrease with increasing temperature on the scale $\hbar / T_{\gamma}$ where $T_{\gamma}$ denotes the traversal time of the orbit. Our results provide a consistent explanation for the quantum oscillations superimposed on the classical magnetoresistivity of antidot lattices recently observed by Weiss et al. [5].

Our approach implies that the quantum oscillations in antidot lattices are closely related to the well-known Shubnikov-de Haas oscillations. We have made this relation explicit by giving an analogous semiclassical treatment of Shubnikov-de Haas oscillations. One finds that the amplitude of Shubnikov-de Haas oscillations is of a lower power in $\hbar$ and hence larger than that of the quantum oscillations in chaotic systems. In this sense, the periodic-orbit contribution to the conductivity is a sensitive probe of quantum chaos. This is analogous to the well-known result that periodic-orbit contributions to the density of states are much larger for integrable systems (Berry-Tabor formula [40]) than for chaotic systems (Gutzwiller formula [24]). Most importantly, the close relationship between Shubnikov-de Haas oscillations and the periodic-orbit contribution in chaotic systems implies that both effects should be observable under analogous conditions. Hence observation of the latter requires that the elastic and inelastic mean free paths be much larger than the length of the periodic orbit but may be smaller than the sample size. Furthermore, the temperature must be smaller than $\hbar / T_{\gamma}$ which is the analog of the Landau-level spacing for Shubnikov-de Haas oscillations. Both effects also require a macroscopically homogeneous sample. Otherwise periodic orbits in different parts of the sample contribute with different phases resulting in a reduced amplitude due to destructive interference.

Our calculation of the periodic-orbit contribution to the conductivity of antidot lattices follows the spirit of but is different in detail from Gutzwiller's derivation of the periodicorbit contributions to the density of states. Like Gutzwiller's trace formula, our results are strictly valid only for completely chaotic systems. Of course, many systems exhibit mixed dynamics with both regular and chaotic regions in phase space. In fact, antidot lattices tend to become mixed systems with increasing magnetic field or increasing steepness of the antidot potential. A quantitative theory for the periodic-orbit contributions to the 
conductivity in mixed systems remains a challenge for future work. Fortunately, the antidot sample which was used to demonstrate the quantum oscillations for the first time [5] appears to be quite close to completely chaotic dynamics over a significant magnetic-field range so that a comparison with our theoretical results is meaningful.

It is interesting to compare our work with other recent semiclassical studies of transport properties. Physically, one also expects a weak-localization correction to the classical conductivity for the antidot lattice. It appears reasonable that the weak-localization correction for antidot lattices should be of the same order in Planck's constant $\hbar$ as that for diffusive systems. This would imply that the weak-localization correction is of the same order in $\hbar$ as the periodic-orbit contributions computed in this paper. Nevertheless, the stationary-phase conditions do not allow for interference between time-reversed trajectories. Recently, Argaman argued in the context of diffusive systems that a resolution of this dilemma requires one to go beyond standard semiclassical theory. For small but finite $\hbar$ he also included trajectories which approximately (but not strictly) satisfy the stationary-phase conditions and was then able to rederive the diagrammatic results for weak localization in the diffusive regime.

Semiclassical expressions for the conductance have also been derived recently starting from the Landauer-Büttiker formula for the two-probe conductance. In particular, semiclassical treatments have been given for weak localization and conductance fluctuations in phase-coherent ballistic microstructures. In this formulation the semiclassical conductance is given in terms of a double sum over classical trajectories entering through one lead and exiting through the other. It is not clear how periodic orbits of the microstructure would enter in this approach. It is clearly desirable to gain a deeper understanding of the relationship between these different approaches to judge conclusively the range of validity of the semiclassical results for transport properties. 


\section{ACKNOWLEDGMENTS}

We thank D. Weiss for making unpublished data available to us, and enjoyed helpful and informative discussions with him and with H. Baranger, E. Doron, K. Ensslin, R. Gerhardts, B. Huckestein, R. Jalabert, K. Richter, S. Tomsovic, H. Weidenmüller, M. Zirnbauer, and W. Zwerger.

\section{APPENDIX A: SEMICLASSICAL GREEN FUNCTION FOR ELECTRONS IN A MAGNETIC FIELD}

In this appendix, we derive the semiclassical Green function in Eq. (6) for an electron in the two-dimensional $x-y$ plane subject to a perpendicular magnetic field. We include a

weak disorder potential resulting in the broadening $\Gamma=\hbar / 2 \tau_{\text {el }}$ of the Green function defined above Eq. (2). We recall that the Green function is the Fourier transform of the propagator,

$$
G_{\mathbf{r}^{\prime}, \mathbf{r}}^{+}(E)=\frac{1}{i \hbar} \int_{-\infty}^{\infty} d t \exp \left\{\frac{i}{\hbar}(E+i \Gamma) t\right\} K\left(\mathbf{r}^{\prime}, \mathbf{r} ; t\right)
$$

where the propagator

$$
K\left(\mathbf{r}^{\prime}, \mathbf{r} ; t\right)=\theta(t)\left\langle\mathbf{r}^{\prime}|\exp (-i \hat{H} t / \hbar)| \mathbf{r}\right\rangle
$$

is known exactly [41],

$$
K\left(\mathbf{r}^{\prime}, \mathbf{r} ; t\right)=\theta(t) \frac{m}{2 \pi i \hbar t} \frac{\omega_{c} t / 2}{\sin \left(\omega_{c} t / 2\right)} \exp \left\{\frac{i m \omega_{c}}{2 \hbar}\left[\frac{\left|\mathbf{r}-\mathbf{r}^{\prime}\right|^{2}}{2} \cot \frac{\omega_{c} t}{2}-\left(x y^{\prime}-x^{\prime} y\right)\right]\right\} .
$$

To simplify notation we denote the phase of $K\left(\mathbf{r}^{\prime}, \mathbf{r} ; t\right)$ by

$$
R\left(\mathbf{r}, \mathbf{r}^{\prime} ; t\right)=\frac{m}{2}\left(\frac{\left|\mathbf{r}-\mathbf{r}^{\prime}\right|^{2}}{2} \cot \frac{\omega_{c} t}{2}-\left(x y^{\prime}-x^{\prime} y\right)\right)
$$

In the semiclassical limit $\hbar \rightarrow 0$ the integral in Eq. (A1) can be performed by the stationaryphase method. The stationary-phase condition reads

$$
E=\frac{m \omega_{c}^{2}}{8}\left(\frac{\left|\mathbf{r}-\mathbf{r}^{\prime}\right|}{\sin \left(\omega_{c} t / 2\right)}\right)^{2}
$$


This condition has no solutions for $\left|\mathbf{r}-\mathbf{r}^{\prime}\right|>2 R_{c}$ reflecting the fact that there are no classical trajectories between two points further apart than twice the cyclotron radius $R_{c}$. Thus the semiclassical $G_{\mathbf{r}^{\prime}, \mathbf{r}}^{+}(E)$ vanishes for $\left|\mathbf{r}-\mathbf{r}^{\prime}\right|>2 R_{c}$. On the other hand, points with $\left|\mathbf{r}-\mathbf{r}^{\prime}\right|<2 R_{c}$ lie on two different cyclotron orbits (cf. Fig. 1), and one finds an infinite set of stationary times $T_{n, q}, n=0,1,2, \ldots, q=S, L$,

$$
T_{n, q}=\frac{2 \pi n}{\omega_{c}}+t_{q}
$$

with

$$
\begin{aligned}
& t_{S}=\frac{2}{\omega_{c}} \arcsin \left(\left|\mathbf{r}-\mathbf{r}^{\prime}\right| / 2 R_{c}\right), \\
& t_{L}=\frac{2}{\omega_{c}}\left[\pi-\arcsin \left(\left|\mathbf{r}-\mathbf{r}^{\prime}\right| / 2 R_{c}\right)\right] .
\end{aligned}
$$

Here $n$ counts the number of complete cyclotron revolutions and $q$ distinguishes between the two orbits. The $T_{n, q}$ are the traversal times for the classical motion from $\mathbf{r}$ to $\mathbf{r}^{\prime}$. Performing the stationary-phase integrals, one obtains

$$
\begin{aligned}
G_{\mathbf{r}^{\prime}, \mathbf{r}}^{+}(E)= & \frac{m}{2 i \hbar}\left(\frac{1}{2 \pi i \hbar}\right)^{1 / 2} \sum_{n=0}^{\infty} \sum_{q=S, L} \frac{\omega_{c}}{\sin \left(\omega_{c} T_{n, q} / 2\right)}\left(\left.\frac{\partial^{2} R}{\partial t^{2}}\right|_{T_{n, q}}\right)^{-1 / 2} \\
& \times \exp \left(-T_{n, q} / 2 \tau_{e l}\right) \exp \left\{\frac{i}{\hbar}\left[E T_{n, q}+R\left(\mathbf{r}, \mathbf{r}^{\prime} ; T_{n, q}\right)\right]\right\}
\end{aligned}
$$

One has

$$
\left.\frac{\partial^{2} R}{\partial t^{2}}\right|_{T_{n, q}}=\omega_{c} E \cot \frac{\omega_{c} T_{n, q}}{2}
$$

and replaces all trigonometric functions in the amplitude by their absolute values. The phases are absorbed into the Maslov indices

$$
\begin{aligned}
& \eta_{n, S}=2 n, \\
& \eta_{n, L}=2 n+1 .
\end{aligned}
$$

The phase appearing in Eq. (A8) is just the action

$$
S\left(\mathbf{r}, \mathbf{r}^{\prime} ; E\right)=E T_{n, q}+\frac{m \omega_{c}}{2}\left[\frac{\left|\mathbf{r}-\mathbf{r}^{\prime}\right|^{2}}{2} \cot \frac{\omega_{c} T_{n, q}}{2}-\left(x y^{\prime}-x^{\prime} y\right)\right],
$$

where the stationary times $T_{n, q}$ depend on $\mathbf{r}, \mathbf{r}^{\prime}$, and $E$ as described by Eqs. (A6, A7). Putting everything together one arrives at the result given in Eq. (6). 


\section{APPENDIX B: STABILITY AMPLITUDE}

In this appendix a derivation of Eq. (34) is presented. First, we note that evaluation of the amplitudes of the Green function (20) in the local coordinate system $(y, z)$ of the trajectory yields [24]

$$
D_{\gamma_{i}}\left(\mathbf{r}, \mathbf{r}^{\prime}\right)=\frac{1}{\sqrt{|\mathbf{v}(z)|\left|\mathbf{v}\left(z^{\prime}\right)\right|}}\left|\frac{\partial^{2} S_{\gamma_{i}}}{\partial y \partial y^{\prime}}\right| .
$$

Hence we need to simplify the expression

$$
a_{\gamma}=\left\{\left|\frac{\partial^{2} S_{\gamma_{1}}}{\partial y \partial y^{\prime}}\right|\left|\frac{\partial^{2} S_{\gamma_{2}}}{\partial y \partial y^{\prime}}\right| /|\operatorname{det} F|\right\}^{1 / 2} .
$$

To do so, we introduce a matrix $A^{(i)}$ for trajectory $\gamma_{i}$ which relates to linear order small transverse deviations in the initial and final momentum $\delta \mathbf{p}^{T}=\left[\delta p, \delta p^{\prime}\right]$ to small transverse deviations in the initial and final positions $\delta \mathbf{y}^{T}=\left[\delta y, \delta y^{\prime}\right]$,

$$
\delta \mathbf{p}=A \delta \mathbf{y} .
$$

In terms of the action $S_{\gamma_{i}}$ the matrix $A^{(i)}$ can be readily expressed as

$$
A^{(i)}=\left[\begin{array}{cc}
-\frac{\partial^{2} S_{\gamma_{i}}}{\partial y^{2}} & -\frac{\partial^{2} S_{\gamma_{i}}}{\partial y \partial y^{\prime}} \\
\frac{\partial^{2} S_{\gamma_{i}}}{\partial y^{\prime} \partial y} & \frac{\partial^{2} S_{\gamma_{i}}}{\partial y^{2}}
\end{array}\right] .
$$

Hence, all quantities appearing in $a_{\gamma}$ can be expressed in terms of matrix elements of $A^{(1)}$ and $A^{(2)}$. It turns out that $a_{\gamma}$ is more conveniently expressed in terms of the monodromy matrices $M$ and $N$ of the trajectories $\gamma_{1}$ and $\gamma_{2}$, respectively. The monodromy matrix relates to linear order the final transverse deviations $\delta \mathbf{x}^{\prime T}=\left[\delta y^{\prime}, \delta p^{\prime}\right]$ to the initial transverse deviations in phase space $\delta \mathbf{x}^{T}=[\delta y, \delta p]$,

$$
\delta \mathbf{x}^{\prime}=M \delta \mathbf{x} .
$$

An important property of the monodromy matrix is that it has unit determinant, $\operatorname{det} M=1$. The matrices $A^{(1)}$ and $A^{(2)}$ can now be easily written in terms of the matrix elements $m_{i j}$ and $n_{i j}$ of the monodromy matrices, respectively. One finds 


$$
A^{(1)}=\left[\begin{array}{cc}
-\frac{m_{11}}{m_{12}} & \frac{1}{m_{12}} \\
m_{21}-\frac{m_{11} m_{22}}{m_{12}} & \frac{m_{22}}{m_{12}}
\end{array}\right] .
$$

and likewise for $A^{(2)}$. Using these relations we find for $a_{\gamma}$ in terms of the monodromy matrices $M$ and $N$,

$$
\begin{aligned}
a_{\gamma}^{2} & =\left|\frac{\left(1 / m_{12}\right)\left(1 / n_{12}\right)}{\left(m_{11} / m_{12}-n_{11} / n_{12}\right)\left(m_{22} / m_{12}-n_{22} / n_{12}\right)-\left(1 / m_{12}-1 / n_{12}\right)^{2}}\right| \\
& =\left|\frac{m_{12} n_{12}}{n_{12}^{2}\left(m_{11} m_{22}-1\right)+m_{12}^{2}\left(n_{11} n_{22}-1\right)-n_{12} m_{12}\left(m_{11} n_{22}+n_{11} m_{22}-2\right)}\right| \\
& =\left|n_{12} m_{21}+m_{12} n_{21}-m_{11} n_{22}-n_{11} m_{22}+2\right|^{-1} \\
& =\left|\operatorname{Tr} M N^{-1}-2\right|^{-1} .
\end{aligned}
$$

Here we used that $\operatorname{det} M=\operatorname{det} N=1$. Finally, we note that $M N^{-1}=M_{\gamma}^{p}$, where $M_{\gamma}$ is the monodromy matrix of the (primitive) periodic orbit. Hence, one finds the result

$$
a_{\gamma}=\left|\operatorname{Tr} M_{\gamma}^{p}-2\right|^{-1 / 2}
$$

which proves Eq. (34). It is remarkable that only the stability of the periodic orbit enters in this factor, regardless of how long the trajectories $\gamma_{1}$ and $\gamma_{2}$ are.

\section{APPENDIX C: TERMS INVOLVING TWO ADVANCED (RETARDED) GREEN FUNCTIONS}

In this appendix it is shown that the contributions to the conductivity (4) involving two advanced or two retarded Green functions vanish in the semiclassical approximation. While we focus on the calculation for antidot lattices, the same statement holds in our treatment of Shubnikov-de Haas oscillations because the factor displayed in (C2) below is also present in

this case. For definiteness, consider the contribution $\sigma_{x x}^{++}$. Using the semiclassical expression for the Green function (19) one has

$$
\begin{aligned}
\sigma_{x x}^{++}=-\frac{i e^{2}}{8 \pi^{2} \hbar^{2} \Omega} \int d^{2} r d^{2} r^{\prime} \sum_{\gamma_{1}\left(\mathbf{r}, \mathbf{r}^{\prime}\right)} & \sum_{\gamma_{2}\left(\mathbf{r}^{\prime}, \mathbf{r}\right)}\left(v_{1}\right)_{x}\left(v_{2}^{\prime}\right)_{x} D_{\gamma_{1}}\left(\mathbf{r}, \mathbf{r}^{\prime}\right) D_{\gamma_{2}}\left(\mathbf{r}^{\prime}, \mathbf{r}\right) \\
& \times \exp \left\{\frac{i}{\hbar}\left[S_{\gamma_{2}}\left(\mathbf{r}, \mathbf{r}^{\prime}\right)+S_{\gamma_{1}}\left(\mathbf{r}^{\prime}, \mathbf{r}\right)\right]-\frac{i \pi}{2}\left[\eta_{\gamma_{2}}+\eta_{\gamma_{1}}\right]\right\} .
\end{aligned}
$$


One notes that in contrast to $\sigma_{x x}^{+-}$this contribution involves both trajectories running from $\mathbf{r}$ to $\mathbf{r}^{\prime}$ and from $\mathbf{r}^{\prime}$ to $\mathbf{r}$. In particular, there is no classical contribution for which the phase factor cancels. Again performing the spatial integrals by the stationary-phase method, one finds that the integrand becomes stationary when $\mathbf{v}_{1}=\mathbf{v}_{2}$ and $\mathbf{v}_{1}^{\prime}=\mathbf{v}_{2}^{\prime}$, where $\mathbf{v}_{i}$ and $\mathbf{v}_{i}^{\prime}$ denote the velocities of $\gamma_{i}$ at position $\mathbf{r}$ and $\mathbf{r}^{\prime}$, respectively. The stationary-phase condition is satisfied when both $\gamma_{1}$ and $\gamma_{2}$ are parts of the same periodic orbit. This is illustrated in Fig. 7. A straight-forward extension of the calculation in appendix B shows that the amplitude factors also combine in this case to yield $\left|\operatorname{Tr} M_{\gamma}^{p}-2\right|^{-1 / 2}$ involving only the monodromy matrix of the periodic orbit. However, the contribution $\sigma_{x x}^{++}$vanishes in the semiclassical limit because of the integrals over $z$ and $z^{\prime}$ along the periodic orbit. The analog of Eq. (35) reads

$$
\exp \left(-p T_{\gamma} / 2 \tau_{e l}\right) \sum_{n=0}^{\infty} \int_{0}^{T_{\gamma}} d t \int_{0}^{T_{\gamma}} d t^{\prime} v_{x}(t) v_{x}\left(t+t^{\prime}\right) \exp \left(-n T_{\gamma} / \tau_{e l}\right)
$$

This expression vanishes because the exponential suppression factor due to disorder does not depend on $t$ or $t^{\prime}$ and hence the integrals are over one period of a periodic function with zero mean. A completely analogous argument can be used to show that $\sigma_{x x}^{--}$vanishes in the semiclassical approximation. 


\section{REFERENCES}

[1] For reviews of quantum chaos see "Chaos and Quantum Physics," ed. by M.-J. Gianonni, A. Voros, and J. Zinn-Justin (Elsevier, Amsterdam, 1991).

[2] S. Washburn and R.A. Webb, Adv. Phys. 55, 1311 (1992).

[3] D. Weiss, M.L. Roukes, A. Menschig, P. Grambow, K. von Klitzing, and G. Weimann, Phys. Rev. Lett. 66, 2790 (1991).

[4] C.M. Marcus, A.J. Rimberg, R.M. Westervelt, P.F. Hopkins, and A.C. Gossard, Phys. Rev. Lett. 69, 506 (1992).

[5] D. Weiss, K. Richter, A. Menschig, R. Bergmann, H. Schweizer, K. von Klitzing, and G. Weimann, Phys. Rev. Lett. 70, 4118 (1993).

[6] M.W. Keller, O. Mittal, and D.E. Prober, Surf. Sci. 305, 501 (1994).

[7] A.M. Chang, H.U. Baranger, L.N. Pfeiffer, and K.W. West, preprint (1994).

[8] L.P. Lévy, D.H. Reich, L. Pfeiffer, and K. West, Physica B 189, 204 (1993).

[9] D. Mailly, C. Chapelier, and A. Benoit, Phys. Rev. Lett. 70, 2020 (1993).

[10] R.A. Jalabert, H.U. Baranger, and A.D. Stone, Phys. Rev. Lett. 65, 2442 (1990).

[11] H.U. Baranger, R.A. Jalabert, and A.D. Stone, Chaos 3, 665 (1993).

[12] H.U. Baranger, R.A. Jalabert, and A.D. Stone, Phys. Rev. Lett. 70, 3876 (1993).

[13] F. von Oppen and E.K. Riedel, Phys. Rev. B 48, 9170 (1993).

[14] F. von Oppen, Ph.D. thesis, University of Washington, 1993; and to appear in Phys. Rev. B.

[15] D. Ullmo, K. Richter, and R.A. Jalabert, preprint (1993).

[16] P.A. Lee, A.D. Stone, and H. Fukuyama, Phys. Rev. B 35, 1039 (1987). 
[17] B.L. Altshuler, Pis'ma Zh. Eksp. Teor. Fiz. 41, 530 (1985) [JETP Lett. 41, 648 (1985)].

[18] For reviews of weak localization in disordered metals see P.A. Lee and T.V. Ramakrishnan, Rev. Mod. Phys. 57, 287 (1985); and G. Bergmann, Phys. Rep. 107, 1 (1984).

[19] K. Nakamura, S. Ishizaka, and F. Nihey, Physica B 197, 155 (1994).

[20] Ya. G. Sinai, Russ. Math. Surv. 25 No. 2, 137 (1970).

[21] An experiment on small phase-coherent antidot lattices has been reported in R. Schuster, K. Ensslin, D. Wharam, S. Kühn, J.P. Kotthaus, G. Böhm, W. Klein, G. Tränkle, and G. Weimann, Phys. Rev. B 49, 8510 (1994). Here we do not consider this regime.

[22] A short account of part of this work was given in G. Hackenbroich and F. von Oppen, preprint (1994).

[23] O. Bohigas, in Ref. [1].

[24] M. Gutzwiller, in Ref. [1].

[25] R. Fleischmann, T. Geisel, and R. Ketzmerick, Phys. Rev. Lett. 68, 1367 (1992).

[26] There has been some controversy over which kind of trajectories are most relevant for the classical transport anomalies. Some investigators favor so-called runaway trajectories: E.M. Baskin, G.M. Gusev, Z.D. Kvon, A.G. Pogosov, and M.V. Entin, JETP Lett. 55, 579 (1992). Here we do not enter into this discussion as it does not seem relevant to the quantum corrections which are at the focus of this paper.

[27] T. Ando, J. Phys. Soc. Japan 37, 1233 (1974).

[28] S. Washburn and R.A. Webb, Adv. Phys. 35, 375 (1986).

[29] Note that the leading Weyl contribution to the density of states is independent of the magnetic field.

[30] There have also been numerical computations of the full quantum mechanics of antidot 
lattices in H. Silberbauer, J. Phys.: Cond. Mat. 4, 7355 (1992); H. Silberbauer and U. Rössler, unpublished. However, the quantum oscillations of the conductivity discussed in this paper are currently outside the reach of these calculations. See also R.B.S. Oakeshott and A. MacKinnon, J. Phys.: Cond. Mat. 5, 6971, (1993); 5, 6991 (1993); and 6, 1519 (1994).

[31] N. Argaman, Y. Imry, and U. Smilansky, Phys. Rev. B 47, 4440 (1993).

[32] O. Agam, J. Phys. I (France) 4, 697 (1994).

[33] S.D. Prado, M.A.M. de Aguiar, J.P. Keating, R. Egydio de Carvalho, preprint (1994).

[34] After completion of this work we learned that a connection between quantum corrections to the conductivity and periodic orbits was briefly mentioned previously in $\mathrm{M}$. Wilkinson, J. Phys. A 20, 2415 (1987), and in B. Eckhardt, S. Fishman, K. Müller, and D. Wintgen, Phys. Rev. A 45, 3531 (1992). While the emphasis in these papers is on formal developments in semiclassical theory, we focus here on the predictions for the physics of ballistic semiconductor structures.

[35] Alternatively, one can compute the ac conductivity and take the zero-frequency limit after the thermodynamic limit. However, this procedure is rarely implemented. When taking the limits in the opposite order (as one does when calculating the dc conductivity directly) without disorder scattering, there can be no dissipation because of the finite level spacing. Disorder averaging avoids this problem since it effectively leads to a continuous spectrum.

[36] P. Strěda, J. Phys. C 15, 717 (1982).

[37] D. Weiss, private communication.

[38] The elastic mean free path for which these numbers were calculated is smaller than the value measured for the unpatterned sample in Ref, [5]. This could be due to additional scattering from the rough edges of the antidots. This effect should be less significant 
for samples with smaller antidot diameter. Indeed, one observes that $\delta \sigma_{x x} \gg \delta \sigma_{x y}$ in such samples consistent with larger values of $\omega \tau_{e l}$ (D. Weiss, private communication). However, one should also keep in mind that this value of $\tau_{e l}$ may be an artifact of our treatment of disorder.

[39] N. Argaman, preprint (1994).

[40] M.V. Berry and M. Tabor, J. Phys. A 10, 371 (1977).

[41] R.P. Feynman and A.R. Hibbs, "Quantum Mechanics and Path Integrals," (McGrawHill, New York, 1965). 


\section{FIGURES}

Fig. 1 Sketch of classical trajectories which contribute to the semiclassical Green function $G_{\mathbf{r}^{\prime}, \mathbf{r}}^{+}(E)$ for electrons in a homogeneous magnetic field. The two shortest trajectories from $\mathbf{r}$ to $\mathbf{r}^{\prime}$ labeled by $\mathrm{S}$ (for short) and L (for long) are depicted by bold lines. There are also contributions from trajectories which complete additional revolutions around the cyclotron orbits of radius $R_{c}$.

Fig. 2 Sketch of classical trajectories $\gamma_{1}$ and $\gamma_{2}$ from $\mathbf{r}$ to $\mathbf{r}^{\prime}$ which satisfy the stationaryphase conditions (29, 30). Both trajectories follow a periodic orbit $\gamma$ (dashed line). The shaded area depicts an antidot.

Fig. 3 Experimental results of Weiss et al. [5. 37] for quantum oscillations $\sigma(T=0.4 \mathrm{~K})-$ $\sigma(T=4.7 \mathrm{~K})$ as function of magnetic field $B$ in longitudinal conductivity $\sigma_{x x}$ (full line) and Hall conductivity $\sigma_{x y}$ (dashed line). The arrows indicate the magnetic-field values where minima in the conductivity are predicted according to Eq. (36).

Fig. 4 Numerical result for classical resistivity of the model potential (52) with $\beta=2$ as function of magnetic field $B$. The resistivity exhibits a single dominant peak at $B=B_{0}$ where the cyclotron orbit equals half the lattice spacing of the antidot arrays. The parameters were chosen according to the experimental parameters for the main sample investigated in Ref. [5] which also showed a single peak in the resistivity at the same magnetic-field value. This supports the choice of $\beta=2$ for the steepness of the antidot potential.

Fig. 5 (a) Poincare surface of section $\left(x / a, v_{x} / \sqrt{E_{F} / m}\right)$ at $y=0(\bmod a)$ with exponent $\beta=2$ for $R_{c}=a / 2$ and antidot diameter $d=a / 2$ appropriate for the experimental sample in Ref. [5]. Within our resolution the classical dynamics is completely chaotic. (b) The same surface of section for $\beta=4$ and $d=a / 3$. Here one finds a mixed phase space with both islands of regular motion and regions of chaotic motion. 
Fig. 6 Contour plot of the antidot potential (thin lines) together with the relevant periodic orbits for $B=B_{0}$ where the cyclotron radius $R_{c}=a / 2$. The innermost lines correspond to the boundaries of the classically-forbidden regions.

Fig. 7 Sketch of classical trajectories $\gamma_{1}$ from $\mathbf{r}^{\prime}$ to $\mathbf{r}$ and $\gamma_{2}$ from $\mathbf{r}$ to $\mathbf{r}^{\prime}$ which satisfy the stationary-phase conditions for the terms involving two advanced or two retarded Green functions. Both trajectories follow a periodic orbit $\gamma$ (dashed line). The shaded area depicts an antidot. 


\section{TABLES}

Table I Numerical results for the traversal times $T_{\gamma}$, velocity correlations $C_{\gamma}\left(v_{x}, v_{x}\right)$, and stabilities $\left|\operatorname{Tr} M_{\gamma}-2\right|^{-1 / 2}$ of the periodic orbits shown in Fig. 6. The parameters are chosen appropriately for the experimental sample in Ref. [5]. The magnetic field corresponds to $R_{c}=a / 2$. For comparison, numbers are also given for the cyclotron orbits of unpatterned samples.

\begin{tabular}{||c|c|c|c|}
\hline \hline Orbit & $T_{\gamma}\left[10^{-12} \mathrm{~s}\right]$ & $C_{\gamma}\left(v_{x}, v_{x}\right)$ & $\left|\operatorname{Tr} M_{\gamma}-2\right|^{-1 / 2}$ \\
\hline$b$ & 3.42 & 1.41 & 0.42 \\
$c_{1}$ & 4.04 & 1.62 & 0.62 \\
$c_{2}$ & 4.07 & 1.63 & 0.46 \\
$c_{3}$ & 4.04 & 1.62 & 0.62 \\
cyclotron & 3,14 & 1.26 & - \\
orbit & & & \\
\hline \hline
\end{tabular}

